\title{
EchoGéo
}

$13 \mid 2010$

Afrique, 50 ans d'indépendance : État et territoires

\section{Portfolio - Regards de géographes sur l'Afrique du Sud pour des passeurs nommés Antheaume}

Introduction

Frédéric Giraut, Sylvain Guyot et Myriam Houssay-Holzschuch

\section{OpenEdition}

Journals

Édition électronique

URL : https://journals.openedition.org/echogeo/12054

DOI : $10.4000 /$ echogeo.12054

ISSN : 1963-1197

Éditeur

Pôle de recherche pour l'organisation et la diffusion de l'information géographique (CNRS UMR 8586)

Référence électronique

Frédéric Giraut, Sylvain Guyot et Myriam Houssay-Holzschuch, «Portfolio - Regards de géographes sur l'Afrique du Sud pour des passeurs nommés Antheaume », EchoGéo [En ligne], 13 | 2010, mis en ligne le 20 septembre 2010, consulté le 03 août 2021. URL : http://journals.openedition.org/echogeo/ 12054 ; DOI : https://doi.org/10.4000/echogeo.12054

Ce document a été généré automatiquement le 3 août 2021

EchoGéo est mis à disposition selon les termes de la licence Creative Commons Attribution - Pas d'Utilisation Commerciale - Pas de Modification 4.0 International (CC BY-NC-ND) 


\section{Portfolio - Regards de géographes sur l'Afrique du Sud pour des passeurs nommés Antheaume}

Introduction

Frédéric Giraut, Sylvain Guyot et Myriam Houssay-Holzschuch

Illustration 1 - Elisabeh Deliry-Antheaume et Benoît Antheaume

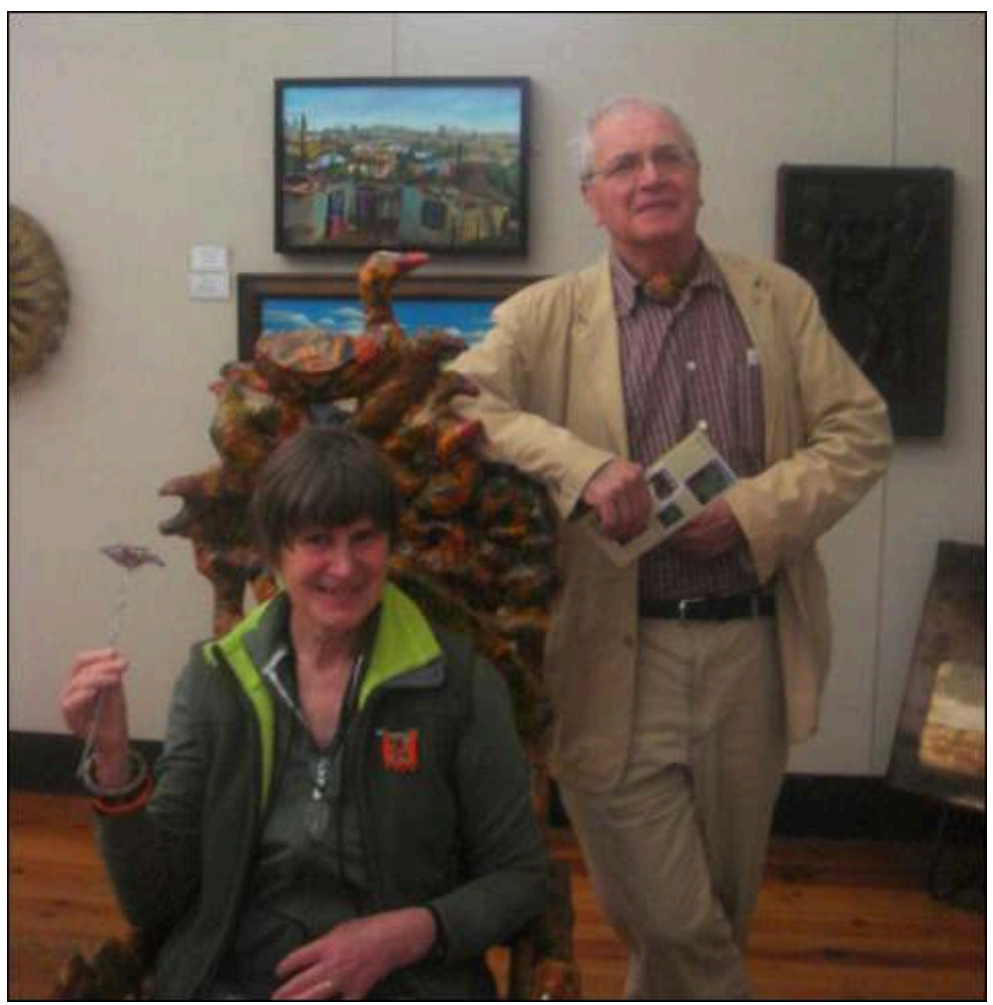

Auteur : Pierre Antoine Landel, Newcastle (Afrique du Sud), 2007. 
1 L'Afrique du Sud post-apartheid au cours des quinze dernières années a été le terrain et le cadre d'une remarquable génération de travaux de géographes (ou assimilés) francophones : juniors (ou ex juniors) qui y ont fait leur thèse, et seniors qui ont mené des programmes en partenariat avec des collègues sud-africains. Un géographe, ex représentant de l'Institut de Recherche pour le Développement en Afrique du Sud, Benoît Antheaume, a été un vrai facilitateur pour la plupart de ces travaux et un passeur pour les échanges avec les géographes sud africains. Benoît Antheaume prenant sa retraite, c'est l'occasion de lui rendre hommage, ainsi qu'à son épouse, Elisabeth Deliry-Antheaume, géographe et photographe, elle aussi véritable passeur par sa connaissance des townships, de leurs habitants et de leurs artistes de murals qu'elle a médiatisé. La forme de ce portfolio centré sur l'image lui doit d'ailleurs beaucoup.

2 À partir de quelques contacts établis dès le début des années 1990 par l'ORSTOM-IRD, et à la faveur de l'installation d'une représentation IRD dans la nouvelle Afrique du Sud, ils ont participé à étendre les réseaux de partenariats notamment chez les géographes, en parallèle avec la mise en place de l'IFAS-Recherche (Institut Français d'Afrique du Sud) dirigé à la même époque par Philippe Gervais-Lambony. Grâce à cette conjonction spatio-temporelle, Benoît Antheaume et Philippe Gervais-Lambony ont, de concert, mis en place les conditions institutionnelles, matérielles, intellectuelles de la recherche francophone en Afrique du Sud en prenant soin qu'elle se développe à la fois en partenariat et dans une atmosphère conviviale, voire amicale, d'échanges. Venus après une longue expérience de terrains africains, au Togo notamment, et des settlerssocieties australes (Nouvelle Zélande, Nouvelle Calédonie), l'investissement des Antheaume s'est effectué sur le plan institutionnel avec une inlassable action de Benoît pour tisser des liens avec de nouveaux collègues sud-africains issus notamment des universités "historiquement défavorisées» de Durban-Westville, de Fort Hare, du KwaZulu (Zululand), du Transkei mais bien vite recrutés par les grandes universités sudafricaines où ils jouent un rôle parfois majeur dans leur discipline. Citons Brij Maharaj de l'Université du KwaZulu-Natal présent dans ce dossier.

3 Cet effort d'ouverture des partenariats s'est prolongé dans les Suds au-delà de la seule Afrique du Sud avec notamment les programmes Corus sur les recompositions territoriales associant le Maroc par ses géographes de l'Université Cadi Ayyad à ceux d'un réseau d'universités sud-africaines et françaises ou encore le programme sur les migrations transnationales porté par des sociologues comme Simon Bekker de Stellenbosch et associant nombre de spécialistes africains.

4 L'investissement s'est fait également sur le terrain, arpenté inlassablement à la recherche des marges sud-africaines, celles des townships, des anciens bantoustans et des zones frontalières. Elisabeth a souvent guidé des chercheurs de tous horizons dans ces townships où elle a suivi des projets de fresques murales communautaires ou individuels : townships ayant pour nom Kliptown à Soweto, Gugulethu au Cap ou encore New Brighton à Port Elizabeth et même d'improbables townships de petites villes comme Makana à Grahamstown ou Esikhawini à Richards Bay.

5 Les 14 contributions ici réunies illustrent la richesse des travaux réalisés sur les transformations spatiales, la réinvention des lieux et les réappropriations symboliques de la nouvelle Afrique du Sud mais également sur l'inertie des structures urbaines et spatiales (Voir localisation sur carte ci-dessous). Elles représentent aussi les générations de chercheurs et les types de partenariats impliqués dans ces travaux ainsi 
que de nouvelles pistes de recherches tracées à partir des chantiers ouverts dans la décennie 1990.

Illustration 2 - Localisation des illustrations présentées dans le portfolio

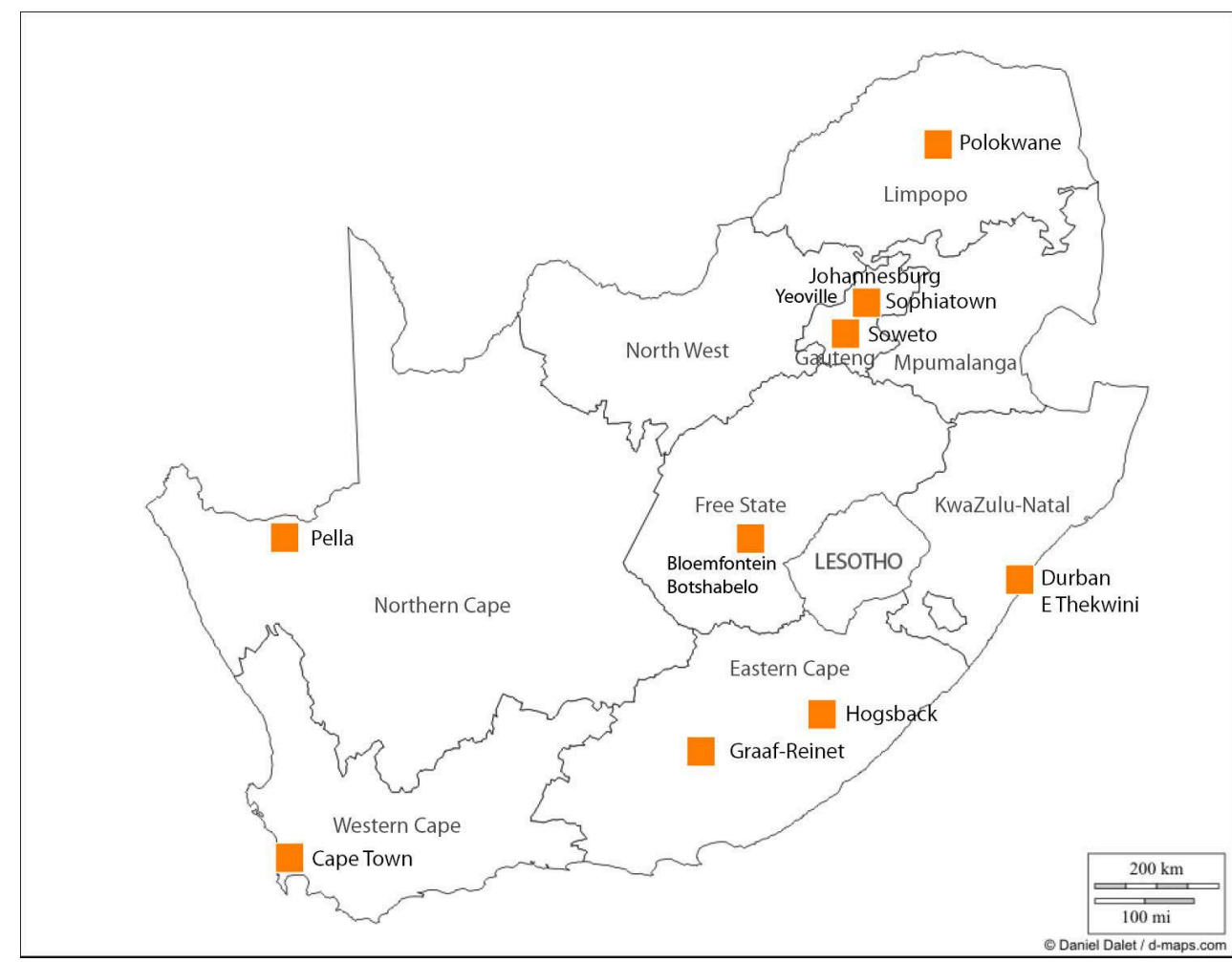

En complément de cette introduction une bibliographie non exhaustive rend compte des thèses francophones soutenues, souvent avec l'appui de l'IFAS et/ou de l'IRD, en géographie durant la période des décennies 1990 et 2000 ainsi que des ouvrages et numéros spéciaux de revues portant sur les mutations des espaces sud-africains ${ }^{1}$.

\section{BIBLIOGRAPHIE}

Thèses soutenues en géographie sur la nouvelle Afrique du Sud (liste indicative) :

Bénit C., 2001. La fragmentation urbaine à Johannesburg. Recomposition des pouvoirs locaux, mobilités de travail et dynamiques résidentielles dans la ville postapartheid. Université de Poitiers.

Blanchon D., 2003. Enjeux territoriaux et impacts environnementaux des transferts d'eau inter-bassin en Afrique australe. Université de Lyon II et de Paris X.

Folio F., 2003. Les villes du KwaZulu-Natal en Afrique du Sud. Université de La Réunion.

Guillaume Ph., 2001. Johannesburg, géographies de l'exclusion. Université de Reims.

Guyot S., 2003. L'environnement contesté : la territorialisation des conflits environnementaux sur le littoral du Kwazulu-natal (Afrique du Sud: KosiBay, St Lucia, Richards Bay et Port Shepstone). Université 
de Paris X-Nanterre. http://tel.archives-ouvertes.fr/index.php?

halsid=2u1qugq03o0bmlr5f4i1harmu3\&view_this_doc=tel-00363411\&version=1

Houssay M., 1997. Le Territoire volé : une géographie culturelle des quartiers noirs de Cape Town. Université de Paris IV-Sorbonne. http://tel.archives-ouvertes.fr/tel-00185377/en/

Mainet H., 2001. Durban : Les Indiens, leurs territoires, leur identité.

Morange M., 2001. Propriétaires et locataires en Afrique du Sud. Politiques publiques et dynamiques résidentielles dans la ville post-apartheid : le cas de Port Elizabeth. Université Paris X-Nanterre.

Vacchiani-Marcuzzo C., 2005. Mondialisation et système de villes : les entreprises étrangères et l'évolution des agglomérations sud-africaines. Université de Paris I. http://tel.archives-ouvertes.fr/ tel-00011351/

Vermeulin S., 2006. Centralités métropolitaines et disparités socio-spatiales, le cas de Durban. Université de Paris X-Nanterre. http://sites.google.com/site/stephanevermeulin/these

\section{Ouvrages :}

Antheaume B. (ed.), 1999. L'Afrique du Sud : recompositions territoriales, problèmes identitaires, migrations de populations et réseaux. L'Espace Géographique, $\mathrm{n}^{\circ} 2$.

Antheaume B. \& Giraut F. (eds.), 2005. Le territoire est mort, Vive les territoires! Paris, IRD Editions.

Antheaume B., Giraut F. \& Maharaj B. (eds.), 2003. Recompositions territoriales, confronter et innover. Actes des rencontres scientifiques franco-sud africaines de l'innovation territoriale / Territorial restructurings, comparisons and innovations. Proceedings of the French-South African meeting on territorial innovation. Grenoble, PACTE/IRD. http://www.pacte.cnrs.fr/spip.php?rubrique173

Benit-Gbaffou C. \& Gervais-Lambony P. (eds), 2008. Les formes de la démocratie locale dans les villes sud-africaines. Revue Tiers Monde, ${ }^{\circ}$ 4. http://www.cairn.info/revue-revue-tiersmonde-2008-4.htm

Benit-Gbaffou C., Fabiyi S. \& Peyroux E. (eds.), 2009. Sécurisation des quartiers et gouvernance locale. Enjeux et défis pour les villes africaines (Afrique du Sud, Kenya, Mozambique, Namibie, Nigeria). Paris, Karthala.

Blanchon D., 2009. L'espace hydraulique sud-africain. Le partage des eaux. Paris, Karthala.

Bouillon A., 1999. L'immigration africaine en Afrique du sud - Les migrants francophones des années 90. Paris, Karthala.

Boujrouf S., Antheaume B., Giraut F. \& Landel P.-A., (eds.), 2009. Les territoires à l'épreuve des normes: référents et innovations, Contributions croisées sud-africaines, françaises et marocaines. Marrakech et Grenoble, Université Cadi Ayyad et Revue Montagnes Méditerranéennes.

Cubizolles S., 2010. Le football en Afrique du Sud. Vécu d'un township au Cap oriental. Paris, Karthala.

Darbon D., 2000. L'après-Mandela : enjeux sud-africains et régionaux. Paris, Karthala.

Dubresson A. \& Jaglin S. (éds.), 2008. Le Cap après l'apartheid. Gouvernance métropolitaine et changement urbain. Paris, Karthala.

Fassin D. (ed.), 2004. Afflictions : L'Afrique du Sud de l'apartheid au sida. Paris, Karthala.

Fauvelle-Aymar F.-X., 2006. Histoire de l'Afrique du Sud. Paris, Seuil.

Gervais-Lambony P., 2003. Territoires citadins, 4 villes africaines. Paris, Belin.

Gervais-Lambony P. (ed.), 1998, L’Afrique du Sud recomposée. Géographie et Culture, nº 28. 
Gervais-Lambony P., 1997. L'Afrique du Sud et les Etats voisins. Paris, Armand Colin.

Gervais-Lambony P., Landy F. \& Oldfield S. (eds.), 2003. Espaces arc-en-ciel. Identités et territoires en Afrique du Sud et en Inde. Paris, Karthala.

Giraut F. \& VacchianiI-Marcuzzo C., 2009. Territories and Urbanisation in South Africa, Atlas and geohistorical information system (DYSTURB). Paris, IRD Editions (Collection Atlas Numérique).

Giraut F. (dir.), 2006. Etude comparée des politiques d'aménagement du territoire et de développement régional dans les pays du sud. Paris, DGCID (Collection études). http://www.diplomatie.gouv.fr/fr/ IMG/pdf/Pubb.pdf

Guillaume P., 2001. Johannesburg, géographies de l'exclusion. Johannesburg/Paris, Ifas-Karthala.

Guillaume P., Pejout N. \& Wa Kabwe-Segatti A., 2004. L'Afrique du Sud 10 ans après : transition accomplie? Johannesburg/Paris, Ifas-Karthala.

Guyot S., Dellier J. (eds.), 2009. Rethinking the Wild Coast (South Africa) : eco-frontiers versus livelihoods in Pondoland. VDM Verlag, Saarbrücken.

Guyot S., 2008. Zulu Shores, South Africa: Green disputes in Black and White. VDM Verlag :

Saarbrücken.

Guyot S., 2006. Rivages zoulous. L'environnement au service du politique en Afrique du Sud. Paris, Karthala.

Houssay-Holzschuch M., 1996. Mythologies territoriales en Afrique du Sud, un essai de géographie culturelle. Paris, Éditions du CNRS.

Houssay-Holzschuch M., 1999. Le Cap, ville sud-africaine : ville blanche, vies noires. Paris, L'Harmattan.

Jaglin S., 2005. Service d'eau en Afrique subsaharienne. La fragmentation urbaine en question. Paris, CNRS éditions.

Jaglin S., Gervais-Lambony P. \& Mabin A., 1999. La question urbaine en Afrique australe. Perspectives de recherche. Paris, IFAS-Karthala.

Mainet-Valeix H., 2002. Durban, les Indiens, leurs territoires, leur identité. Paris, IFAS- Karthala.

Morange M., 2006. La question du logement à Mandela-City (ex Port Elizabeth). Paris, Karthala.

\section{NOTES}

1. On consultera aussi avec profit la liste des travaux plurisiciplinaires sur l'Afrique du Sud compilée par l'IFAS, http://www.ifas.org.za/research/.

\section{AUTEURS}

\section{FRÉDÉRIC GIRAUT}

Université de Genève, Frederic.Giraut@unige.ch 


\section{SYLVAIN GUYOT}

Université de Limoges, sylvain.guyot@unilim.fr

MYRIAM HOUSSAY-HOLZSCHUCH

Université de Lyon, ENSL \& IUF, Myriam.Houssay-Holzschuch@ens-lyon.fr 